\title{
Endotoxemia in Critically III Patients with COVID-19
}

\author{
Sobia Khan ${ }^{\mathrm{a}}$ Olena Bolotova ${ }^{\mathrm{a}}$ Haseena Sahib $^{\mathrm{a}}$ Debra Foster $^{\mathrm{b}}$ \\ Sandeep K. Mallipattu ${ }^{\mathrm{a}, \mathrm{c}}$ \\ aDepartment of Medicine, Stony Brook University, Stony Brook, NY, USA; bSpectral Medical Inc., Toronto, ON, \\ Canada; 'Renal Section, Northport VA Medical Center, Northport, NY, USA
}

\section{Keywords}

Endotoxemia · Endotoxin activity assay · COVID-19 .

SARS-CoV-2 - Acute kidney injury

\begin{abstract}
Introduction: Mechanism(s) mediating critical illness in coronavirus disease 2019 (COVID-19) remain unclear. Previous reports demonstrate the existence of endotoxemia in viral infections without superimposed gram-negative bacteremia, but the rate and severity of endotoxemia in critically ill patients with COVID-19 requires further exploration. $\mathbf{M a}$ terials and Methods: This is a single-center cross-sectional study of 92 intensive care unit patients diagnosed with COVID-19 pneumonia. Endotoxin activity (EA) was measured in patients that met the following criteria: (1) age $\geq 18$ years and (2) multi-organ dysfunction score $>9$ from March 24, 2020, to June 20, 2020. Results: A total of 32 patients met the inclusion/exclusion criteria for measurement of EA. The median age of the study cohort was 60 years with a majority male $(21 / 32,65 \%)$ with hypertension (50\%). A significant proportion of the patients exhibited either elevated EA in the intermediate range $(0.40-0.59$ EA units) $(10 / 32,31 \%)$ or high range ( $\geq 0.60$ EA units) $(14 / 32,44 \%)$ or were nonresponders
\end{abstract}

(C) 2021 S. Karger AG, Basel www.karger.com/bpu
(NRs, low neutrophil response) to EA $(6 / 32,19 \%)$, with the presence of gram-negative bacteremia only in 2/32 (6\%) patients. Low EA was reported in $2 / 32$ patients. NRs $(5 / 6,83 \%)$ and patients with high $\mathrm{EA}(7 / 14,50 \%)$ exhibited higher acute kidney injury (AKI) as compared to patients with low/intermediate EA level (1/12, 8.3\%). Discussion/Conclusion: Elevated EA was observed in a large majority of critically ill patients with COVID-19 and multi-organ dysfunction despite a low incidence of concurrent gram-negative bacteremia. While we observed that elevated EA and nonresponsiveness to EA were associated with AKI in critically ill patients with COVID-19, these findings require further validation in larger longitudinal cohorts.

(c) 2021 S. Karger AG, Basel

\section{Introduction}

Coronavirus disease 2019 (COVID-19) has been declared a global pandemic. As of February 20, 2020, >100 million confirmed cases resulting in over 2 million deaths were reported worldwide [1]. In early reports from China, a significant proportion of patients demonstrated features of pneumonia in the setting of COVID-19 and ap- 
proximately a $3 \mathrm{rd}$ of those cases required admission to the intensive care unit (ICU) with $15 \%$ being fatal [2]. These initial reports also noted that critical disease, which included respiratory failure, septic shock and/or multi-organ failure occurred in 5\% of the cases [3]. Specifically, septic shock was present in $6.4 \%$ patients with severe COVID-19, but blood cultures and lower respiratory cultures were negative for bacteria or fungus in $76 \%$ of these patients $[4,5]$.

Endotoxin (lipopolysaccharide) is a component of the outer wall of gram-negative bacteria, which has been investigated and acknowledged as one of the triggers of lethal shock during sepsis and drivers of cytokine storm [6]. Furthermore, the Multi-Center Endotoxin Detection in Critical Illness (MEDIC) study has demonstrated that high endotoxin activity (EA) level in blood was associated with higher rates of sepsis and mortality [7]. Monti et al. [8] also showed that high EA level in patients with sepsis was associated with higher vasopressor requirements and increased hospital mortality as compared with intermediate and low EA groups. While elevation in EA occurs as a result of gram-negative bacteremia, high EA levels have also been reported to occur independent of gram-negative bacteremia $[9,10]$, suggesting the potential for endotoxin translocation across a dysfunctional gastrointestinal barrier. Furthermore, ischemia-mediated gut barrier dysfunction contributing to organ failure, in the absence of primary gastrointestinal injury, has been supported by experimental models [11].

Human pathogenic coronaviruses, SARS-CoV and SARS-CoV-2, gain entry into target cells by binding to the angiotensin-converting enzyme-2 (ACE-2) receptor, which is expressed in endothelial cells as well as epithelial cells of the lung, intestine, and kidneys [12]. Specifically, enterocytes in ileum and colon express the ACE-2 receptor [13]. Therefore, there is a possibility that breakdown in the intestinal barrier in the setting of severe $\mathrm{CO}$ VID-19 might lead to endotoxin translocation. A recent case series from Thailand shows that bacterial toxins and DNA are present in patients with COVID-19, but without gram-negative bacteremia [14]. It has also been shown that adverse ventilation strategy can cause pulmonary to systemic translocation of endotoxins [15]. In addition, the inflammatory response to COVID-19 could have been augmented by a 2 nd hit, such as mechanical ventilation $(\mathrm{MV})$ or inflammatory response to trauma $[9,10]$. Furthermore, during the previous $\mathrm{H} 1 \mathrm{~N} 1$ and $\mathrm{H} 5 \mathrm{~N} 1$ viral pandemics, endotoxemia was reported in the absence of gram-negative bacteremia [16]. In this single-center, observational study, we measured EA in adult critically ill patients with COVID-19 and multi-organ dysfunction, and report our clinical end points based on patient demographics, presenting clinical characteristics and outcomes relative to EA level.

\section{Materials and Methods}

\section{Study Design and Participants}

This is a single-center, cross-sectional study in hospitalized patients with COVID-19 that were admitted to Stony Brook University Hospital ICU from March 24, 2020 to June 20, 2020. To determine the critically ill patients with COVID-19 that might be at risk for endotoxemia, we utilized the multi-organ dysfunction score (MODS) $>9$ from the EUPHRATES clinical study to measure EA [17]. The aim of this study was to determine the rate and severity of endotoxemia in critically ill patients with COVID-19 admitted to the ICU. Our inclusion criteria were as follows: (1) hospitalized patients with positive reverse transcriptase polymerase chain reaction for SARS-CoV-2 collected by nasopharyngeal swab, (2) age $\geq 18$ years, (3) requirement of ICU admission, and (4) MODS $>9$. Exclusion criteria were as follows: (1) patients with MODS $<9$, (2) end-stage kidney disease requiring chronic dialysis, and (3) patient with severe congestive heart failure (NYHA class IV, Ejection fraction $<35 \%)$. Blood EA was measured in patients who met the inclusion and exclusion criteria using the previously described endotoxin activity assay (EAA) (Spectral Diagnostics, Toronto, Canada) [7]. This study was approved by the Stony Brook University Institutional Review Board (IRB2020-00474).

\section{Data Collection and Definition of Variables}

Demographics, vital signs, laboratory values, and radiographic tests were extracted from electronic health records. Demographics included age, gender, race, ethnicity, and reported comorbidities such as hypertension, diabetes, coronary artery disease, chronic kidney disease, chronic obstructive pulmonary disease (COPD) as documented by the healthcare provider's note, and ICD-9 and ICD-10 codes collectively. Vital signs included blood pressure with daily average systolic and diastolic blood pressure and ratio of $\mathrm{PaO}_{2}$ to $\mathrm{FiO}_{2}\left(\mathrm{PaO}_{2} / \mathrm{FiO}_{2}\right)$ on the day of the endotoxin assay, and maximum temperature within $48 \mathrm{~h}$ of the EA level. Laboratory data included serum creatinine ( $\mathrm{sCr}$ ), alanine aminotransaminase, aspartate aminotransferase, and procalcitonin levels on the day of the EA measurement. Inflammatory markers included D-dimer, lactate dehydrogenase, ferritin, c-reactive protein, erythrocyte sedimentation rate were extracted on the day of admission, peak values during hospitalization, and day of the EA measurement. Blood culture results were collected within $48 \mathrm{~h}$ of the EA measurement.

Radiologic assessment included chest x-ray or CT to confirm the diagnosis of COVID-19 pneumonia. Clinical outcomes included the incidence of acute kidney injury (AKI), need for renal replacement therapy (RRT), incidence of MV, need for vasopressor support, duration of MV, length of hospitalization, and outcome of hospitalization (discharged alive or death).

COVID-19 pneumonia was defined by positive reverse transcriptase polymerase chain reaction for SARS-CoV-2 collected by nasopharyngeal swab and evidence of lung opacities as documented by the radiologist. AKI was defined as an increase in $\mathrm{sCr} \geq 0.3$ $\mathrm{mg} / \mathrm{dL}$ within $48 \mathrm{~h}$ period or an increase in $\mathrm{sCr} 1.5$ times baseline 


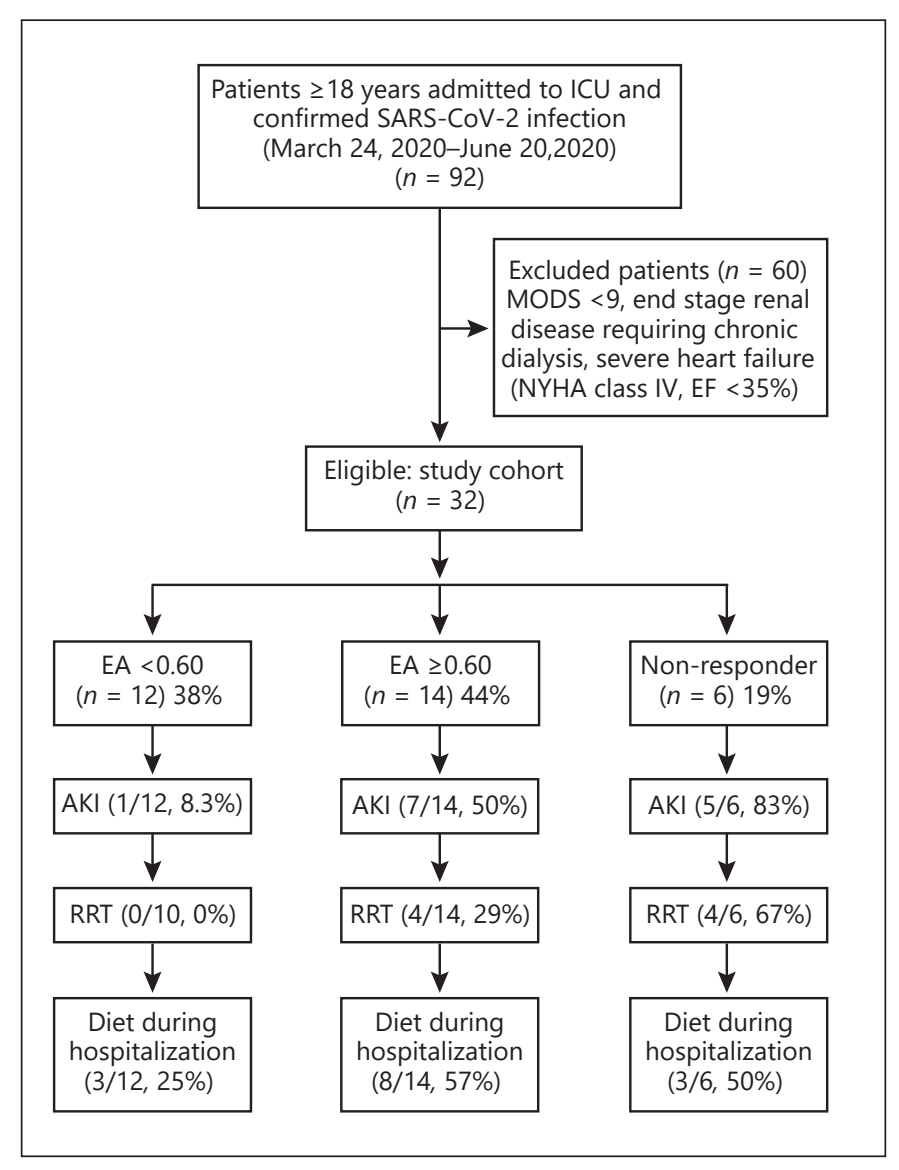

Fig. 1. Flow chart of selection of study cohort that had EA level measured. Total number and percentage of patients in intermediate, high, and NR EA groups is shown. Total number and percentage of patients with AKI, required RRT, and that died is shown per each group. EA, endotoxin activity; AKI, acute kidney injury; RRT, renal replacement therapy; NR, nonresponder; ICU, intensive care unit; MODS, multi-organ dysfunction score.

within last 7 days or urine output $<0.5 \mathrm{~mL} / \mathrm{kg} / \mathrm{h}$ for $6 \mathrm{~h}$ as per KDIGO guidelines [18].

Assay for EA is approved by the US Food and Drug Administration as a highly sensitive and specific rapid diagnostic test for endotoxemia [7]. As per manufacturer protocol and as previously described [7, 19], $3 \mathrm{~mL}$ of whole blood was collected in a standard collection tube containing EDTA anticoagulant. The assay measures the chemiluminescent reaction from anti-LPS antibody-antigen complex to activated neutrophils and a lumiphor to create emission of light. The amount of chemiluminescence is proportional to the logarithmic concentration of LPS in the sample and is specific to the lipid moiety of LPS of gram-negative bacteria. The reported EA level is a relative scale from 0 to $1.0 \mathrm{E}$ A units based on an endotoxin standard that is added to each patient sample and was initially categorized into low, high, and nonresponder (NR) groups as previously described [7]. Intermediate EA is defined from 0.40 to 0.59 units and represents an elevated risk of severe sepsis. High EA is defined as $\geq 0.60$ units and represents high risk of severe sepsis. NR is a test result obtained from patients whose neutrophils do
Table 1. Demographics and clinical characteristics of the study cohort

\begin{tabular}{lc}
\hline Demographics and clinical characteristics & $N=32$ \\
\hline Age & $60 \pm 15.8$ \\
Gender, $n(\%)$ & $21(65)$ \\
$\quad$ Male & $11(35)$ \\
Female & \\
Race/ethnicity, $n(\%)$ & $0(0)$ \\
Black & $13(40)$ \\
White & $3(10)$ \\
Asian & $16(50)$ \\
Other & $16(50)$ \\
Hispanic & $16(50)$ \\
Comorbidities, $n(\%)$ & $7(22)$ \\
HTN & $2(6)$ \\
DM & $2(6)$ \\
CKD & $1(3)$ \\
CAD & $7^{\mathrm{b}}(29)$ \\
COPD & 2 \\
Positive blood cultures $( \pm 48 \text { h of test day })^{\mathrm{a}}$ & 4 \\
Gram-negative bacteremiac & 2 \\
Gram-positive bacteremia & \\
Fungemia &
\end{tabular}

HTN, hypertension; DM, diabetes; CAD, coronary artery disease; CKD, chronic kidney disease; COPD, chronic obstructive pulmonary disease. ${ }^{\text {a }}$ Blood cultures available for $24 / 32$ patients $\pm 48 \mathrm{~h}$ of test day. ${ }^{\mathrm{b}}$ One patient had positive blood cultures for 2 organisms Candida parapsilosis and Pseudomonas aeruginosa. ${ }^{\mathrm{c}}$ One patient with Klebsiella pneumoniae, 1 patient with Pseudomonas aeruginosa. ${ }^{\mathrm{d}}$ Two patients with Staphylococcus epidermidis, 1 patient with both Staphylococcus hominis and Staphylococcus epidermidis, 1 patient with Enterococcus faecalis group D. ${ }^{\mathrm{e}}$ Two patients with fungemia (Candida parapsilosis and Candida lusitaniae).

not have the ability to respond to preformed immune complexes to EA [7]. The normal reference range was $<0.4$ EA units for the assay and has been previously validated using a healthy population of 43 men and 57 women aged $32 \pm 14$ years [7].

\section{Statistical Analysis}

Statistical analysis was performed with Prism 8 and SPSS. Continuous variables with normal distribution were presented as mean $\pm \mathrm{SD}$, and non-normally distributed variables are presented as mean \pm interquartile range (IQR). Categorical variables presented as numbers and percentages. Normally distributed continuous variables were compared with ordinary one-way ANOVA test between $>2$ groups and Welch's $t$ test for 2 groups of independent variables. Non-normally distributed continuous variables were analyzed with Kruskal-Wallis test when compared between $>2$ groups and Mann-Whitney test when compared between 2 groups of independent variables. Categorical variables were compared with Fisher's exact test. Statistical significance was defined as $p$ value $<0.05$. 
Table 2. Clinical characteristics, laboratory values, and clinical outcomes in the study cohort

\begin{tabular}{|c|c|c|c|c|c|c|}
\hline \multirow{2}{*}{$\begin{array}{l}\text { Clinical characteristics, laboratory } \\
\text { values, and clinical outcomes }\end{array}$} & \multirow{2}{*}{$\begin{array}{l}\mathrm{EA}<0.6 \\
(N=12)\end{array}$} & \multirow{2}{*}{$\begin{array}{l}\mathrm{EA} \geq 0.6 \\
(N=14)\end{array}$} & \multirow{2}{*}{$\begin{array}{l}\mathrm{NR} \\
(N=6)\end{array}$} & \multicolumn{3}{|l|}{$p$ value } \\
\hline & & & & $\begin{array}{l}\mathrm{EA} \geq 0.6 \\
\text { vs. } \mathrm{EA}<0.6\end{array}$ & $\begin{array}{l}\mathrm{EA} \geq 0.6 \\
\text { vs. NR }\end{array}$ & $\begin{array}{l}\text { EA }<0.6 \\
\text { vs. NR }\end{array}$ \\
\hline \multicolumn{7}{|l|}{ Demographics } \\
\hline Age, years & $55.8 \pm 20$ & $63.7 \pm 13$ & $60.7 \pm 12.3$ & 0.26 & 0.63 & 0.54 \\
\hline Male & $9(75 \%)$ & $9(64 \%)$ & $4(66 \%)$ & $>0.99$ & $>0.99$ & $>0.99$ \\
\hline \multicolumn{7}{|l|}{ Comorbidities } \\
\hline HTN & $5(42 \%)$ & $9(64 \%)$ & $2(33 \%)$ & 0.43 & 0.34 & $>0.99$ \\
\hline $\mathrm{DM}$ & $3(25 \%)$ & $4(29 \%)$ & 0 & $>0.99$ & 0.27 & 0.51 \\
\hline CKD & 0 & $2(14 \%)$ & 0 & 0.48 & $>0.99$ & $>0.99$ \\
\hline CAD & $1(8 \%)$ & $1(7 \%)$ & 0 & $>0.99$ & $>0.99$ & $>0.99$ \\
\hline COPD & 0 & 0 & $1(16 \%)$ & $>0.99$ & 0.3 & 0.33 \\
\hline \multicolumn{7}{|l|}{ Vital signs } \\
\hline Temperature, $\operatorname{Tmax} \pm 48 \mathrm{~h},{ }^{\circ} \mathrm{C}$ & $38.2 \pm 0.9$ & $38.5 \pm 0.4$ & $37.8 \pm 0.9$ & 0.39 & 0.09 & 0.28 \\
\hline $\mathrm{PaO}_{2} / \mathrm{FiO}_{2}$ ratio & $168 \pm 84$ & $220 \pm 90$ & $161 \pm 113$ & 0.14 & 0.29 & 0.90 \\
\hline \multicolumn{7}{|l|}{ Blood pressure } \\
\hline SBP, mm Hg & $119 \pm 12$ & $126 \pm 12$ & $115 \pm 17$ & 0.09 & 0.18 & 0.58 \\
\hline DBP, mm Hg & $61 \pm 6$ & $64 \pm 9$ & $55 \pm 9$ & 0.22 & 0.05 & 0.14 \\
\hline MAP, $\mathrm{mm} \mathrm{Hg}$ & $81 \pm 7$ & $86 \pm 8$ & $75 \pm 10$ & 0.13 & 0.02 & 0.16 \\
\hline SOFA score (test day) & $7.3 \pm 2.7$ & $7.1 \pm 3.5$ & $10.0 \pm 3.9$ & 0.88 & 0.15 & 0.17 \\
\hline \multicolumn{7}{|l|}{ Laboratory values (test day) } \\
\hline $\mathrm{WBC}, \mathrm{K} / \mu \mathrm{L}$ & $15.3 \pm 9.2$ & $17.9 \pm 13.9$ & $17.6 \pm 8.1$ & 0.70 & 0.60 & 0.68 \\
\hline $\mathrm{sCr}, \mathrm{mg} / \mathrm{dL}^{\mathrm{a}}$ & $0.4(0.2-0.7)$ & $0.8(0.4-1.8)$ & $1.5(0.8-2.3)$ & 0.16 & 0.64 & 0.09 \\
\hline $\mathrm{eGFR}, \mathrm{mL} / \mathrm{min} / 1.73 \mathrm{~m}^{2}$, a & $120(98-120)$ & $87(28-120)$ & $59(27-90)$ & 0.06 & 0.60 & 0.07 \\
\hline AST, IU/L & $45(26-1,246)$ & $28(20-51)$ & $41(16-1,807)$ & 0.12 & 0.75 & 0.60 \\
\hline ALT, IU/L & $38(16-662)$ & $28(11-46)$ & $36(12-470)$ & 0.28 & 0.40 & 0.87 \\
\hline \multicolumn{7}{|l|}{ Inflammatory markers } \\
\hline \multicolumn{7}{|l|}{ Ferritin, ng/mL } \\
\hline Admission & $920(327-1,408)$ & $963(615-1,978)$ & $879(678-2,624)$ & 0.65 & 0.92 & 0.65 \\
\hline Peak & $1,957(1,345-5,507)$ & $2,570(1,419-14,287)$ & $3,934(1,435-20,821)$ & 0.35 & 0.75 & 0.39 \\
\hline Test day & $676(466-1,344)$ & $883(584-1,973)$ & $905(436-2,119)$ & 0.32 & 0.79 & 0.80 \\
\hline \multicolumn{7}{|l|}{$\mathrm{LDH}, \mathrm{IU} / \mathrm{L}$} \\
\hline Admission & $508(262-614)$ & $417(324-704)$ & $408(405-501)$ & 0.94 & 0.95 & 0.37 \\
\hline Peak & $803(668-1,626)$ & $1,005(573-1,598)$ & $847(750-4,370)$ & 0.69 & 0.52 & 0.49 \\
\hline Test day & $433(266-967)$ & $383(311-658)$ & $361(268-674)$ & $>0.99$ & 0.77 & 0.74 \\
\hline \multicolumn{7}{|l|}{$\mathrm{ESR}, \mathrm{mm} / \mathrm{h}$} \\
\hline Admission & $37(16-66)$ & $50(35-59)$ & $81(47-106)$ & 0.58 & 0.11 & 0.09 \\
\hline Peak & $85(64-106)$ & $108(100-127)$ & $130(82-136)$ & 0.07 & 0.85 & 0.19 \\
\hline Test day & $52(13-84)$ & $53(30-86)$ & $60(46-64)$ & 0.60 & 0.98 & 0.67 \\
\hline \multicolumn{7}{|l|}{ D-dimer, ng/mL } \\
\hline Admission & $332(254-601)$ & 317 (196-986) & $384(307-1,840)$ & 0.87 & 0.44 & 0.34 \\
\hline Peak & $6,821(2,256-13,775)$ & $4,380(1,491-12,486)$ & $6,844(2,909-9,493)$ & 0.60 & 0.55 & 0.96 \\
\hline Test day & $940(598-8,087)$ & $906(528-1,901)$ & $1,679(650-3,327)$ & 0.54 & 0.24 & 0.68 \\
\hline \multicolumn{7}{|l|}{ CRP, mg/dL } \\
\hline Admission & $10(2-26)$ & $17(11-24)$ & $21(15-29)$ & 0.09 & 0.30 & 0.16 \\
\hline Peak & $29(13-32)$ & $30(21-39)$ & $41(25-46)$ & 0.31 & 0.48 & 0.21 \\
\hline Day of test & $5(3-10)$ & $6(3-10)$ & $9(1-23)$ & 0.88 & 0.75 & 0.55 \\
\hline Procalcitonin (test day), ng/mL & $0.2(0.1-1.2)$ & $0.6(0.2-1.6)$ & $0.7(0.2-1.2)$ & 0.41 & 0.91 & 0.40 \\
\hline Blood culture & & & & & & \\
\hline Positive blood cultures ( \pm 48 h of test day) & $2(17 \%)^{b}$ & $4(29 \%)^{\mathrm{c}, \mathrm{d}}$ & $1(17 \%)^{e}$ & 0.39 & $>0.99$ & $>0.99$ \\
\hline Gram-negative bacteremia ${ }^{\mathrm{f}}$ & 0 & 2 & 0 & & & \\
\hline Gram-positive bacteremiag & 2 & 1 & 1 & & & \\
\hline Fungemia ${ }^{\mathrm{h}}$ & 0 & 2 & 0 & & & \\
\hline Clinical interventions \& outcomes & & & & & & \\
\hline Vasopressor support & $9(75 \%)$ & $8(57 \%)$ & $6(100 \%)$ & 0.43 & 0.12 & 0.52 \\
\hline Antibiotics & $9(75 \%)$ & $13(93 \%)$ & $3(50 \%)$ & 0.31 & 0.06 & 0.34 \\
\hline Remdesivir & $4(33 \%)$ & $5(36 \%)$ & $1(17 \%)$ & $>0.99$ & 0.60 & 0.61 \\
\hline Corticosteroids & $8(67 \%)$ & $13(93 \%)$ & $5(83 \%)$ & 0.15 & 0.52 & 0.62 \\
\hline Anticoagulation & $11(92 \%)$ & $13(93 \%)$ & $6(100 \%)$ & $>0.99$ & $>0.99$ & $>0.99$ \\
\hline MV & $11(92 \%)$ & $14(100 \%)$ & $6(100 \%)^{\mathrm{i}}$ & 0.46 & $>0.99$ & $>0.99$ \\
\hline Duration, days & $38 \pm 32$ & $33 \pm 28$ & $49 \pm 27$ & $>0.99$ & 0.15 & 0.62 \\
\hline
\end{tabular}


Table 2 (continued)

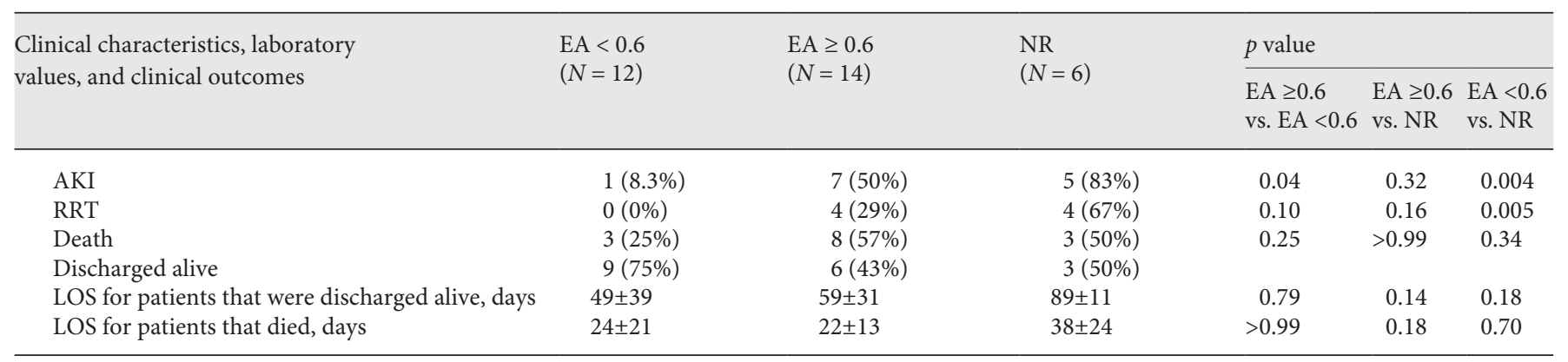

Corticosteroids were counted for patients who received dexamethasone, prednisone, methylprednisolone, and hydrocortisone before and during the test day. Anticoagulation was counted for patients who received therapeutic doses of heparin, lovenox, and apixaban on the test day. Categorical variables are presented as numbers and percentages, normally distributed continuous variables presented as mean $\pm \mathrm{SD}$, and non-normally distributed continuous variables presented as median (25th-75th percentile). COPD, chronic obstructive pulmonary disease; EA, endotoxin activity; CAD, coronary artery disease; CKD, chronic kidney disease; DM, diabetes; $\mathrm{HTN}$, hypertension; $\mathrm{PaO}_{2} / \mathrm{FiO}_{2}$ ratio, ratio of arterial oxygen partial pressure $(\mathrm{PaO}$ in $\mathrm{mm} \mathrm{Hg})$ to fractional inspired oxygen $\left(\mathrm{FiO}_{2}\right)$ expressed as a fraction; SBP, systolic blood pressure; DBP, diastolic blood pressure; MAP, mean arterial pressure; SOFA score, sequential organ failure assessment score; WBC, white blood count; sCr, serum creatinine; eGFR, estimated glomerular filtration rate; AST, aspartate aminotransferase; ALT, alanine aminotransferase; LDH, lactate dehydrogenase; ESR, erythrocyte sedimentation rate; CRP, C-reactive protein; AKI, acute kidney injury; LOS, length of stay; RRT, renal replacement therapy; MV, mechanical ventilation; NR, nonresponder. ${ }^{\mathrm{a}}$ Calculated for the patients who did not require RRT. ${ }^{b}$ Blood cultures available for $10 / 12$ patients. ${ }^{\mathrm{c}}$ Blood cultures available for $10 / 14$ patients. ${ }^{\mathrm{d}}$ One patient was positive for both Pseudomonas aeruginosa and Candida parapsilosis. ${ }^{\mathrm{e}}$ Blood cultures available on $5 / 6$ patients. ${ }^{\mathrm{f}} \mathrm{Gram}$-negative bacteremia due to Pseudomonas aeruginosa and Klebsiella pneumoniae. One patient was positive for both Pseudomonas aeruginosa and Candida parapsilosis. ${ }^{\mathrm{g}}$ Gram-positive bacteremia due to Staphylococcus epidermidis, Staphylococcus hominis, Staphylococcus epidermidis, and Enterococcus faecalis group D. ${ }^{\mathrm{h}}$ Fungemia due to Candida lusitaniae and Candida parapsilosis. One patient was positive for both Pseudomonas aeruginosa and Candida parapsilosis. ${ }^{\mathrm{i}}$ One still mechanically ventilated at the time of discharge.

\section{Results}

A total of 92 patients were screened and 32 patients met the inclusion/exclusion criteria (shown in Fig. 1). The mean age of the participants was $60 \pm 16$ years with the majority male $21 / 32(65 \%)$ (Table 1$)$. Low EA $(<0.4)$ was observed in $2 / 32(6 \%)$ patients, intermediate EA $(0.40-0.59)$ in $10 / 32(31 \%)$ patients, high EA ( $\geq 0.6) 14 / 32$ (44\%) patients, and 6/32 (19\%) patients were classified as NR.

Since there were only 2 patients in the low EA group, we categorized the cohort into 3 groups $(<0.6, \geq 0.6$, and $\mathrm{NR}$ ). All 3 groups were found to have high oxygen requirements with $\mathrm{PaO}_{2} / \mathrm{FiO}_{2}$ ratio of $<300$, with $168 \pm 84$ in the $\mathrm{EA}<0.6$ group, $220 \pm 90$ in the $\mathrm{EA} \geq 0.6$ group, and $161 \pm 113$ in the NR group (Table 2). Procalcitonin levels were elevated in the EA $\geq 0.6$ group, $0.6(0.2-1.6)$, and NR group, 0.7 (0.2-1.2), as compared to EA $<0.6$ group, 0.2 (0.1-1.2), but did not reach statistical significance. Blood culture results were available for $24 / 32$ patients. In these patients, $7 / 24$ had positive blood cultures and only $2 / 7$ patients had gram-negative bacteremia with concurrent fungemia in one of these patients. The remaining positive cultures were a result of gram-positive bacteremia (4/7) and fungemia (1/7). Furthermore, $78 \%$ of the patients were on antibiotics, with a majority on cefepime/vanco- mycin and 5\% of the patients also received meropenem, ertapenem, or linezolid. There were no significant differences in therapies for COVID-19 between the groups (Table 2).

The incidence of AKI was higher in NR group (5/6, $83 \%)$ and the $\mathrm{EA} \geq 0.6$ group $(7 / 14,50 \%)$ than in the EA $<0.6$ group $(1 / 12,8.3 \%), p=0.04$ and $p=0.004$, respectively. The need for RRT was also higher in NR group $(4 / 6,67 \%)$ and the EA $\geq 0.6$ group $(4 / 14,29 \%)$, with none of the patients in the EA $<0.6$ group requiring RRT, $p=$ 0.005 (Table 2).

Of the 32 patients, 31 patients received MV and by the end of the study period, $100 \%$ reached a disposition of discharged alive $(14 / 32,44 \%)$ or death $(18 / 32,56 \%)$. NR $(3 / 6,50 \%)$ and the $\mathrm{EA} \geq 0.6(8 / 14,57 \%)$ groups trended toward a higher mortality than the EA $<0.6$ group ( $3 / 12$, $25 \%)$, but did not reach statistical significance.

\section{Discussion/Conclusion}

In this study, endotoxemia was observed in $75 \%$ of our critically ill patients with COVID-19, with only 2 patients having positive blood cultures for gram-negative organisms. An increase in systemic endotoxin levels can result from infection with gram-negative bacte- 
ria or when the intestinal tract is compromised, causing translocation of gram-negative bacterial products, including endotoxins, into the bloodstream. It has been observed that endotoxemia can occur independent of gram-negative bacteremia $[10,20]$, suggesting a "leaky" or dysfunctional barrier might contribute to endotoxin translocation. This also provides evidence that the intestinal tract might act as an important reservoir of endotoxins in septic shock and multi-organ failure, independent of gram-negative bacteremia. Enterocytes in ileum and colon express the ACE-2 receptor, serving a port of SARS-CoV-2 entry, which subsequently might contribute to a dysfunctional intestinal barrier leading to endotoxin translocation and systemic toxicity [13], leading to a possibility that endotoxin translocation from the intestinal tract might exacerbate the severity of COVID-19.

Among the critically ill, sepsis is a common cause of AKI, but the mechanism by which endotoxemia leads to AKI is incompletely understood. Systemic hypotension, direct renal vasoconstriction, activation of vasoactive hormones, and activation of inflammatory pathways by endotoxins might all contribute to sepsis-associated AKI [21]. The release of endotoxin into the circulatory system is a well-documented phenomenon that occurs during cardiopulmonary bypass procedure and after its discontinuation [9], which likely originates from increased endothelial permeability following ischemia and reperfusion of the gut. Increased EA has been previously associated with renal dysfunction in the setting of infection and sepsis involving altered glomerular filtration, renal tubular damage, and inflammation, leading to cell apoptosis [21]. While the incidence of AKI in patients with COVID-19 and multi-organ dysfunction has been reported to be high in several studies in the USA [22-24], mechanisms mediating this process remain unclear. Our study demonstrates that in critically ill patients with COVID-19 and multi-organ dysfunction, endotoxemia might contribute to the development of AKI.

Similar to previous reports of poor clinical outcomes in NR due to low neutrophil priming response to maximal stimulation with LPS-anti-LPS antibody complexes in EAA [20], we observed that NR were associated with higher incidence of AKI. This poor neutrophil responsiveness might be an indicator of a late anergic state due to prolonged or exaggerated early pro-inflammatory phase [20], suggesting a dysfunctional inflammatory response in a subset of patients with COVID-19 and multiorgan dysfunction.
Our study has some limitations. This is a single-center, cross-sectional study with relatively small cohort of 32 adult ICU patients with COVID-19 that met the criteria of MODS $>9$. While we observed an association between elevated EA levels and AKI in these patients with COVID-19 and severe multi-organ dysfunction, future studies will need to focus on whether endotoxemia can prognosticate the development of AKI in all hospitalized patients with COVID-19 prior to the development of multi-organ dysfunction. For instance, the MEDIC study examined EA levels in patients admitted to the ICU [7], but it has yet to be determined whether elevated EA on admission to hospital has similar prognostic implications. Another limitation of the study was that a majority of the patients were already in their 2 nd month of admission at the time of the test and were, hence, already on broad spectrum antibiotics, which might confound the detection of bacteremia in those patients. However, previous studies report no significant increase in EA levels after initiation of antibiotic therapy [25], but future studies are required to evaluate the impact of antibiotics on EA assay in the setting of COVID-19. In addition, further studies will be required to measure $16 \mathrm{~S}$ rRNA gene sequencing to determine whether the circulating bacteriomes as well as intestinal microbiota might be the source of endotoxemia in these critically ill patients with COVID-19.

In conclusion, we demonstrate that a significant proportion of critically ill patients with COVID-19 and multi-organ dysfunction exhibit endotoxemia independent of gram-negative bacteremia. Future studies are needed to demonstrate the mechanism(s) by which endotoxin levels increase in COVID-19 as well as large prospective studies to determine whether the presence of endotoxemia prognosticates the development of AKI in critically ill patients with and without COVID-19.

\section{Acknowledgment}

We thank Chris Gordon at Stony Brook University for assisting in the EAA.

\section{Statement of Ethics}

The research was conducted ethically in accordance with the World Medical Association Declaration of Helsinki. Informed consent was waived in this study and approved by the Stony Brook University Institutional Review Board (IRB2020-00474). 


\section{Conflict of Interest Statement}

D.F. is an employee of Spectral Medical Inc.

\section{Funding Sources}

Spectral Inc. provided complimentary endotoxin activity assay kits for the study. S.K.M. is supported by funds from NIH/NIDDK (DK112984, DK121846) and Veterans Affairs (I01BX003698, IS1BX004815).

\section{Author Contributions}

S.K., O.B., and S.K.M. designed the study; S.K. and O.B. carried out the data extraction; S.K., O.B., H.S., and S.K.M. analyzed the data; S.K., O.B., and S.K.M. made the tables; S.K., O.B., and S.K.M. drafted the paper; all authors revised the paper; all authors approved the final version of the manuscript.

\section{Data Availability Statement}

All data generated or analyzed during this study are included in this article. Further enquiries can be directed to the corresponding author.

\section{References}

1 Dong E, Du H, Gardner L. An interactive web-based dashboard to track COVID-19 in real time. Lancet Infect Dis. 2020 May;20(5): 533-4.

2 Huang C, Wang Y, Li X, Ren L, Zhao J, Hu Y, et al. Clinical features of patients infected with 2019 novel coronavirus in Wuhan, China. Lancet. 2020 Feb 15;395(10223):497-506.

$3 \mathrm{Wu} \mathrm{Z,} \mathrm{McGoogan} \mathrm{JM.} \mathrm{Characteristics} \mathrm{of} \mathrm{and}$ Important Lessons From the Coronavirus Disease 2019 (COVID-19) Outbreak in China: summary of a report of 72314 cases from the Chinese Center for Disease Control and Prevention. JAMA. 2020 Apr 7;323(13): 1239-42.

4 Guan WJ, Ni ZY, Hu Y, Liang WH, Ou CQ, $\mathrm{He}$ JX, et al. Clinical characteristics of coronavirus disease 2019 in China. N Engl J Med. 2020 Feb 28;382:1708-20.

5 Zhou F, Yu T, Du R, Fan G, Liu Y, Liu Z, et al. Clinical course and risk factors for mortality of adult inpatients with COVID-19 in Wuhan, China: a retrospective cohort study. Lancet. 2020 Mar 11;395(10229):1054-62.

6 Gomez H, Kellum JA. Sepsis-induced acute kidney injury. Curr Opin Crit Care. 2016 Dec; 22(6):546-53.

7 Marshall JC, Foster D, Vincent JL, Cook DJ, Cohen J, Dellinger RP, et al. Diagnostic and prognostic implications of endotoxemia in critical illness: results of the MEDIC study. J Infect Dis. 2004 Aug 1;190(3):527-34.

8 Monti G, Bottiroli M, Pizzilli G, Minnini M, Terzi V, Vecchi I, et al. Endotoxin activity level and septic shock: a possible role for specific anti-endotoxin therapy? Contrib Nephrol. 2010;167:102-10
9 Paternoster G, Rubino G, Balducci A, Maiorano R, Brienza N. Association between endotoxin activity and acute kidney injury in cardiac patients undergoing cardiopulmonary bypass. Blood Purif. 2014;37(1):27-32.

10 Charbonney E, Tsang JY, Li Y, Klein D, Duque P, Romaschin A, et al. Endotoxemia following multiple trauma: risk factors and prognostic implications. Crit Care Med. 2016 Feb;44(2):335-41.

11 Bischoff SC, Barbara G, Buurman W, Ockhuizen T, Schulzke JD, Serino M, et al. Intestinal permeability: a new target for disease prevention and therapy. BMC Gastroenterol. 2014 Nov 18;14:189.

12 Zou X, Chen K, Zou J, Han P, Hao J, Han Z. Single-cell RNA-seq data analysis on the receptor ACE2 expression reveals the potential risk of different human organs vulnerable to 2019-nCoV infection. Front Med. 2020 Apr; 14(2):185-92.

13 Zhang H, Li HB, Lyu JR, Lei XM, Li W, Wu $\mathrm{G}$, et al. Specific ACE2 expression in small intestinal enterocytes may cause gastrointestinal symptoms and injury after 2019-nCoV infection. Int J Infect Dis. 2020 Jul;96:19-24.

14 Sirivongrangson P, Kulvichit W, Payungporn S, Pisitkun T, Chindamporn A, Peerapornratana S, et al. Endotoxemia and circulating bacteriome in severe COVID-19 patients. Intensive Care Med Exp. 2020 Dec 7;8(1):72.

15 Murphy DB, Cregg N, Tremblay L, Engelberts D, Laffey JG, Slutsky AS, et al. Adverse ventilatory strategy causes pulmonary-to-systemic translocation of endotoxin. Am J Respir Crit Care Med. 2000 Jul;162(1):27-33.

16 Liu Q, Zhou YH, Yang ZQ. The cytokine storm of severe influenza and development of immunomodulatory therapy. Cell Mol Immunol. 2016 Jan;13(1):3-10.
17 Dellinger RP, Bagshaw SM, Antonelli M, Foster DM, Klein DJ, Marshall JC, et al. Effect of targeted polymyxin B hemoperfusion on 28 day mortality in patients with septic shock and elevated endotoxin level: the EUPHRATES randomized clinical trial. JAMA. 2018 Oct 9;320(14):1455-63.

18 Khwaja A. KDIGO clinical practice guidelines for acute kidney injury. Nephron Clin Pract. 2012;120(4):c179-84.

19 Cruz DN, Antonelli M, Fumagalli R, Foltran F, Brienza N, Donati A, et al. Early use of polymyxin $B$ hemoperfusion in abdominal septic shock: the EUPHAS randomized controlled trial. JAMA. 2009 Jun 17;301(23):2445-52.

20 Romaschin AD, Klein DJ, Marshall JC. Bench-to-bedside review: clinical experience with the endotoxin activity assay. Crit Care. 2012 Dec 3;16(6):248.

21 Prowle JR. Sepsis-associated AKI. Clin J Am Soc Nephrol. 2018 Feb 7;13(2):339-42.

22 Arentz M, Yim E, Klaff L, Lokhandwala S, Riedo FX, Chong M, et al. Characteristics and outcomes of 21 critically Ill patients with COVID-19 in Washington State. JAMA. 2020 Mar 19;323(16):1612-4

23 Hirsch JS, Ng JH, Ross DW, Sharma P, Shah $\mathrm{HH}$, Barnett RL, et al. Acute kidney injury in patients hospitalized with COVID-19. Kidney Int. 2020 Jul;98(1):209-18

24 Richardson S, Hirsch JS, Narasimhan M, Crawford JM, McGinn T, Davidson KW, et al. Presenting characteristics, comorbidities, and outcomes among 5700 patients hospitalized with COVID-19 in the New York City Area. JAMA. 2020 Apr 22;323(20):2052-9.

25 Mignon F, Piagnerelli M, Van Nuffelen M, Vincent JL. Effect of empiric antibiotic treatment on plasma endotoxin activity in septic patients. Infection. 2014 Jun;42(3):521-8. 